\title{
An Asymptotically Optimal Real-Time Locking Protocol for Clustered Scheduling under Suspension-Aware Analysis
}

\author{
Björn B. Brandenburg \\ Max Planck Institute for Software Systems (MPI-SWS) \\ bbb@mpi-sws.org
}

\section{OPTIMAL LOCKING PROTOCOLS}

The purpose of real-time locking protocols is to limit priority inversions [5], which, intuitively, occur when a high-priority task is delayed by a lower-priority task. Such locking-related delay, also called priority inversion blocking (pi-blocking), is problematic in real-time systems because it can result in deadline misses. However, some pi-blocking is unavoidable when using locks and thus must be bounded and accounted for during schedulability analysis.

Clearly, an "optimal" locking protocol should minimize pi-blocking to the extent possible. Formally, a locking protocol is asymptotically optimal if it ensures that, for any task set, maximum pi-blocking is bounded within a constant factor of the minimal pi-blocking unavoidable in some task set [3]. Interestingly, there exist two classes of schedulability analysis that yield different lower bounds: under suspension-oblivious (s-oblivious) analysis, $\Omega(m)$ pi-blocking is fundamental, whereas under suspension-aware (s-aware) analysis, $\Omega(n)$ pi-blocking is unavoidable in the general case [2, 3], where $m$ and $n$ denote the number of processors and tasks, respectively. As the names imply, the key difference is that suspensions are accounted for explicitly under s-aware analysis, whereas they are (pessimistically) modeled as execution in the s-oblivious case.

For the simpler s-oblivious case, asymptotically optimal locking protocols have been designed for partitioned, global, and clustered job-level fixed-priority ${ }^{1}$ (JLFP) scheduling [4]. The s-aware case, however, is much less understood: only two asymptotically optimal protocols for partitioned JLFP scheduling are known so far $[2,3]$.

In contrast, the problem of optimal s-aware locking under global and clustered JLFP scheduling has remained open to date. While it was initially assumed [3] that Block et al.'s Flexible Multiprocessor Locking Protocol (FMLP) [1]—which is based on $O(n)$ FIFO queues - is asymptotically optimal under global scheduling, it was later observed [2] that this holds only under some, but not all global JLFP schedulers. In fact, it was shown that both priority inheritance [5] and (unconditional) priority boosting [5], one of which is used in each previously proposed s-aware protocol to expedite the completion of critical sections by temporarily raising the effective priority of lock-holding jobs, can give rise to non-optimal $\Omega(\Phi)$ pi-blocking [2], where $\Phi$ is the ratio of the longest and the shortest period (and unbounded in general). Finally, to the best of our knowledge, no asymptotically optimal s-aware locking protocol for the general case of clustered JLFP scheduling has been proposed in prior work.

\footnotetext{
${ }^{1}$ The class of job-level fixed-priority schedulers includes both classic fixedpriority and EDF scheduling. Clustered scheduling is a generalization of both partitioned and global scheduling under which disjoint clusters of processors are scheduled globally.
}

\section{THE GENERALIZED FMLP ${ }^{+}$}

We have solved the problem of asymptotically optimal s-aware locking under clustered JLFP scheduling by devising a new progress mechanism that circumvents the $\Omega(\Phi)$ bound mentioned above.

Priority boosting/inheritance is susceptible to $\Omega(\Phi)$ pi-blocking because a high-priority job $J_{h}$ can be repeatedly preempted by critical sections that were started after $J_{h}$ was already scheduled [2]. This is avoided by the following restricted boosting mechanism. Let $t_{r}\left(J_{i}\right)$ denote the latest point in time that a job $J_{i}$ either (i) was released, (ii) resumed from a locking-unrelated self-suspension, or (iii) requested (i.e., tried to lock) a resource. A priority-boosted, lower-priority job $J_{l}$ may preempt a higher-priority, un-boosted job $J_{h}$ only if $t_{r}\left(J_{l}\right)<t_{r}\left(J_{h}\right)$. This implies that $J_{h}$ is preempted only by critical sections that were in progress when $J_{h}$ became available for scheduling, of which there are at most $n-1=O(n)$ (i.e., one per task, assuming tasks are sequential). Further, it can be shown that lock-holder progress is guaranteed in the sense that at least one lock-holder is always scheduled (if any exist). By scheduling priority-boosted jobs in order of increasing $t_{r}$ timestamps (i.e., FIFO w.r.t. lock request time), $O(n)$ pi-blocking per request is achieved.

Restricted boosting generalizes the idea underlying the partitioned FIFO Multiprocessor Locking Protocol $\left(\mathrm{FMLP}^{+}\right.$) [2], namely to order lock-holding jobs by request time. Combined with $O(n)$ FIFO queues, we obtain a locking protocol that is asymptotically optimal under clustered (and hence also under global) JLFP scheduling.

\section{OUTLOOK}

We believe the proposed protocol offers improved schedulability, in particular if $\Phi$ is large, and are in the process of deriving fine-grained (i.e., non-asymptotic) pi-blocking bounds. We plan to implement and evaluate the protocol in LITMUS ${ }^{\mathrm{RT}}$ and expect overheads to be relatively low due to the simplicity of FIFO queuing and timestampbased preemption checks. Further, we will explore the potential of an analogously designed "restricted inheritance" mechanism.

\section{References}

[1] A. Block, H. Leontyev, B. Brandenburg, and J. Anderson. A flexible real-time locking protocol for multiprocessors. In Proc. RTCSA, 2007.

[2] B. Brandenburg. Scheduling and Locking in Multiprocessor Real-Time Operating Systems. PhD thesis, The University of North Carolina at Chapel Hill, 2011.

[3] B. Brandenburg and J. Anderson. Optimality results for multiprocessor real-time locking. In Proc. RTSS, 2010.

[4] B. Brandenburg and J. Anderson. The OMLP family of optimal multiprocessor real-time locking protocols. Design Automation for Embedded Systems, to appear, 2012.

[5] R. Rajkumar. Synchronization In Real-Time Systems-A Priority Inheritance Approach. Kluwer Academic Publishers, 1991. 